\title{
Is the early or delayed remote ischemic preconditioning the more effective from a microcirculatory and histological point of view in a rat model of partial liver ischemia-reperfusion? ${ }^{1}$
}

\author{
Zsuzsanna Magyar', Gabor Varga", Anita Mester", Souleiman Ghanem", Viktoria Somogyi"', Bence \\ Tanczos $^{\prime V}$, Adam Deak ${ }^{\mathrm{V}}$, Laszlo Bidiga ${ }^{\mathrm{VI}}$, Katalin Peto ${ }^{\mathrm{VII}}$, Norbert Nemeth ${ }^{\mathrm{VIII}}$ \\ 'MD, Department of Operative Techniques and Surgical Research, Institute of Surgery, Faculty of Medicine, University \\ of Debrecen, Hungary. Technical procedures, analysis and interpretation of data, manuscript preparation and writing. \\ "MD, Department of Operative Techniques and Surgical Research, Institute of Surgery, Faculty of Medicine, University of \\ Debrecen, Hungary. Technical procedures, acquisition of data. \\ '"MSc, Department of Operative Techniques and Surgical Research, Institute of Surgery, Faculty of Medicine, University \\ of Debrecen, Hungary. Technical procedures, acquisition of data.

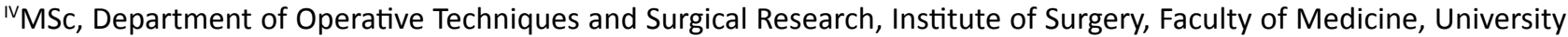 \\ of Debrecen, Hungary. Acquisition of data, statistical analysis. \\ ${ }^{V}$ DVM, PhD, Department of Operative Techniques and Surgical Research, Institute of Surgery, Faculty of Medicine, \\ University of Debrecen, Hungary. Anesthesia, acquisition of data. \\ V'MD, Department of Pathology, Faculty of Medicine, University of Debrecen, Hungary. Histopathological examinations. \\ VIIPhD, Department of Operative Techniques and Surgical Research, Institute of Surgery, Faculty of Medicine, University \\ of Debrecen, Hungary. Conception and design of the study, critical revision. \\ VIIIPhD, DSc, Department of Operative Techniques and Surgical Research, Institute of Surgery, Faculty of Medicine, \\ University of Debrecen, Hungary. Conception and design of the study, critical revision, final approval.
}

\begin{abstract}
Purpose: To compare early- and late-effect remote ischemic preconditioning (RIPC) by analysing the microcirculatory, hemodynamic and histological changes in partial liver ischemia-reperfusion of rats.

Methods: 60-minute partial liver ischemia followed by 120-minute reperfusion was performed without (Control group, $n=7$ ) or with preconditioning. In RIPC groups a tourniquet was applied around the left thigh using 3 cycles of 10-minute ischemia/10-minute reperfusion, one (RIPC$1, n=7$ ) or twenty-four hours (RIPC-24, $n=7$ ) before I/R. Hemodynamic and microcirculatory measurements were performed before and after ischemia and in 30th, 60th and 120th minute of reperfusion and histological examination at the end of reperfusion.

Results: Blood pressure decreased in all groups followed by biphasic changes in Control group. In RIPC groups R120 values returned almost to normal. Heart rate increased in Control and RIPC-1 groups at R120, while RIPC-24 did not show significant changes. Microcirculation of non-ischemic liver stayed constant in Control and showed significant changes in RIPC-24 group, while in ischemic liver elevated by R120 in all groups. RIPC didn't reduce histological alterations.

Conclusion: Considering the survival and the results, both remote ischemic preconditioning protocols had beneficial effect in hepatic ischemia-reperfusion, however the histopathological findings were controversial.

Key words: Ischemia. Reperfusion. Ischemic Preconditioning. Microcirculation. Histology. Liver. Rats.
\end{abstract}




\section{- Introduction}

During liver resections or liver transplantation intraoperative blood loss is a major problem. A common strategy to avoid it is clamping of the portal triad (Pringle manoeuvre) which may lead to ischemia-reperfusion injury of the liver ${ }^{1,2}$. It is well known that ischemia in itself may cause serious damage, however, the restoration of blood flow exacerbates these changes further. The phenomenon is called ischemia-reperfusion injury (IRI) and may contribute to increased postoperative mortality and morbidity following liver surgery ${ }^{3,4}$.

Ischemia-reperfusion injury is a complex, multifactorial pathophysiologic process. It causes direct cellular damage and delayed dysfunction through the activation of inflammatory pathways ${ }^{5}$ but the oxidative stress also contributes to organ dysfunction by several mechanisms.

Previous studies have shown that the hepatic microcirculation is a major target of hepatic $|R|^{6}$. Therefore monitoring the microcirculatory conditions is inevitable in the detection and prediction of organ failure caused by IRI.

Many pharmacological agents and surgical interventions were developed and tested to decrease the ischemia-reperfusion injury ${ }^{7,8}$. Most of these studies have been performed in experimental animals only. Until now only two surgical methods such as intermittent clamping (IC) and ischemic preconditioning (IPC) have been introduced into clinical practice $e^{9-11}$.

IC means intermittent vascular inflow occlusion with short periods of reperfusion in between. The concept of IPC was first introduced by Murry et al. ${ }^{12}$. The procedure consists of brief periods of ischemiareperfusion prior to sustained ischemia. IPC is considered to be an adaptive pathophysiologic phenomenon and has been shown to reduce hepatic ischemia-reperfusion injury in both experimental and clinical studies ${ }^{9}$. IPC may be applied directly or remotely. While there are contradictory data about the favourable application of IC, IPC is a technique that seems to be safe and feasible in the clinical practice too ${ }^{9}$. The disadvantage of the method is the direct stress to the organ and the mechanical trauma to the vessels ${ }^{13}$.

The concept was developed further by demonstrating that brief periods of ischemia and reperfusion applied on a remote organ or tissue can be associated with decreased damage of the target organ. The method known as remote ischemic preconditioning (RIPC) was originally developed by Przyklenk et al. ${ }^{14}$. Numerous studies have described the beneficial, protective effect of remote ischemic preconditioning regarding several organs, including the liver ${ }^{15-17}$. It was successfully applied not only in experimental studies but in clinical conditions too, however, it is still not clarified what the optimal duration and timing (number of cycles, early or late) of the process is.

The current study was designed to compare the effect of early and late remote ischemic preconditioning protocol on microcirculatory and hemodynamic changes caused by partial liver ischemia-reperfusion in the rat. Histological examination of the liver may provide further information for better understanding the pathophysiological changes and how RIPC influences the severity of the damage caused by ischemia-reperfusion.

\section{- Methods}

\section{Experimental animals, operative techniques and sampling protocol}

All procedures were approved and registered by the University of Debrecen Committee of Animal Welfare (permission 
Nr.: 25/2016. UDCAW) in accordance with national and EU regulations [Hungarian Animal Protection Act (Law XVIII/1998) and Directive 2010/63/EU].

The experiment was done using twentyone healthy, mature Crl: WI outbred rats (Toxi-

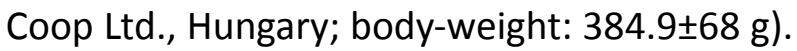
The age of the animal can be a crucial factor in surgical interventions. Older rats are more sensitive for fat accumulations ${ }^{18}$, therefore young animals from the same age (14-16 weeks old) were selected. The rats were randomly allocated to three experimental groups, each group contained 5 male and 2 female rats. As analgesia, Flunixin (2.5 mg/kg s.c.) was administrated one day before the operation to all animals.

The preconditioning procedures and the surgical interventions were performed in general anesthesia using sodium-thiopental (Thiopental: $60 \mathrm{mg} / \mathrm{kg}$ intraperitoneally, supplemented when necessary). The animals were placed in supine position on a heating pad to maintain body-temperature.

In both remote ischemicpreconditioning groups a tourniquet was applied in three cycles by a metal based suture material around the left thigh above the inguinal ligament to occlude the complete hind-limb blood supply. The RIPC protocol included $3 \times 10$ minutes ischemia interspersed with 10-minute reperfusion periods, 1 hour (RIPC-1 group: $n=7$ ) or 24 hours (RIPC-24 group: $n=7$ ) before the sustained ischemia.

In each group the left inguinal region and the abdomen of the experimental animals were completely shaved and disinfected with Betadine. In the left inguinal region a $1 \mathrm{~cm}$ long horizontal incision was performed and after smooth preparation the femoral artery was cannulated. Laparotomy was performed via a midline incision, the intestine was placed gently on the left side of the animal and was covered by a plastic bag to avoid dehydration.

After exposing the organ partial liver ischemia was achieved by using atraumatic microvascular clip on the portal vein and proper hepatic artery above the caudate process and right anterior lobe for 60 minutes to provide $70 \%$ partial liver ischemia ${ }^{19,20}$. Using this model circulation of the intact lobes is preserved, while the above mentioned lobes are excluded from the liver's blood-flow. After removing the microsurgical clip 120 minutes reperfusion period was observed. During the 60-minute ischemic and 120-minute reperfusion period the anterior abdominal wound was covered with two layers, as a first layer a plastic bag was used to minimize evaporative loss and as a second layer a gauze sponge was placed on the abdomen.

Respiratory rate, rectal temperature, liver surface temperature, hemodynamic and microcirculatory measurements were performed before the operation, at the $60^{\text {th }}$ minute of the ischemia (I-60) and at the $30^{\text {th }}$, $60^{\text {th }}$ and $120^{\text {th }}$ minute of the reperfusion ( $R-30$, $\mathrm{R}-60, \mathrm{R}-120)$.

At the end of the follow-up period and the tissue harvest, the animals were euthanized by exsanguination under anesthesia.

\section{Hemodynamic measurements}

For indirect monitoring of blood pressure (systolic, diastolic, MAP) and heart rate Apollo non-invasive Blood Pressure Amplifier (IITC Inc., CA, US) was applied. This measurement technique uses a cuff in which the tail of the experimental animal is inserted.

\section{Respiratory rate}

For following the general state of the experimental animal during the operation and the ischemia-reperfusion period the respiratory rate was observed and documented. 


\section{Rectal temperature}

Rectal temperature measurement was performed by conventional digital thermometer as a common accepted and easy method for monitoring the body temperature in rodents.

\section{Liver surface temperature}

The liver surface temperature was measured at two different points, on the left lateral (ischemic-liver) and right lateral lobe (non-ischemic-liver), using an infrared thermometer.

\section{Microcirculatory measurements}

Laser Doppler flowmetry is a noninvasive, widely used technique to monitor the fluctuation in the microcirculation and the viability of the tissue. The method is based on the Doppler frequency shift phenomenon. The emitted, coherent light show changes in wavelength when it meets moving particles, but it stays unchanged when it enters static tissue elements. The laser Doppler flowmeter emits the laser beam which is reflected from the surface of the moving red blood cells, then the shift in the light is plotted on the computer's screen ${ }^{21}$.

Laser Doppler (LD) flowmetry (LD-01 Laser Doppler Tissue Flowmeter Experimetria Ltd., Hungary) was used for monitoring the changes in the microcirculation. By using a standard pencil probe (MNP100XP, Oxford Optronix Ltd., UK) the microcirculation of the ischemic (Left lateral lobe) and non-ischemic (Right lateral lobe) part of the liver, the kidney and the intestine were detected. During the measurement it is necessary to stabilize the signal to determine the BFU (blood flux unit) of the examined 20 -second period. The findings of the microcirculatory pattern were registered and analysed ${ }^{22}$.

\section{Histopathological examination}

At the end of the observed I/R period samples were collected from the identical signs of the left lateral lobe of the liver, kidney, small-intestine and lung for histopathological examination. The excised tissue samples were fixed in $10 \%$ formaldehyde then were sent to the Department of Pathology, where they were embedded in paraffin and $4 \mathrm{~mm}$ serial sections were performed. Hematoxylin and eosin ( $H$ \& $E$ ) and PAS staining were used to detect and analyse the morphological changes caused by the $I / R$ injury and the intergroup differences after the applied preconditioning protocol. For evaluation of the severity of $I / R$ caused damage Suzuki's score was used ${ }^{23}$. Three different characteristic histological signs (sinusoidal congestion, necrosis, vacuolization) of $\mathrm{I} / \mathrm{R}$ injury were individually graded on a scale from 0 to 4 by an experienced pathologist.

\section{Statistical analysis}

Alldataare expressedasmean \pm standard deviation (S.D.). For the simple comparison of intergroup changes Student's $t$-test or MannWhitney rank sum test, while changes during the examined period at the observed timepoints were determined by one-way ANOVA methods (Bonferroni's or Dunn's test), depending on the data distribution. Significance was assigned a $p$ value of $<0.05$.

\section{- Results}

\section{Hemodynamic measurements}

In the Control group the systolic blood pressure showed a continuous decrease at the end of the ischemic period and at the $30^{\text {th }}$ minute of reperfusion, then the value elevated approximately to the same level of the base value. By R120 it dramatically decreased again and this alteration was significant versus the 
base value of the Control group $(p=0.022$ vs. Base). The systolic blood pressure level of both preconditioning group was lower compare to the Control group at the beginning of the operation, then moderately decreased, however by the $120^{\text {th }}$ minute of the reperfusion the values exceeded the systolic blood pressure of the Control group (Figure 1).

The alterations of the heart rate were fluctuated moderately in the Control group during the observed period, and the level diminished significantly at the $60^{\text {th }}$ minute of the reperfusion compare to the base values $(p=0.045$ vs. Base). In the RIPC- 1 group the heart rate decreased at the $30^{\text {th }}$ and $60^{\text {th }}$ minute of the reperfusion and by the end of the reperfusion elevated again. The heart rate counts of the RIPC-24 group were approximately equal during the experimental protocol (Figure 1).

In spite of the observed alterations there were no statistically significant intergroup differences.
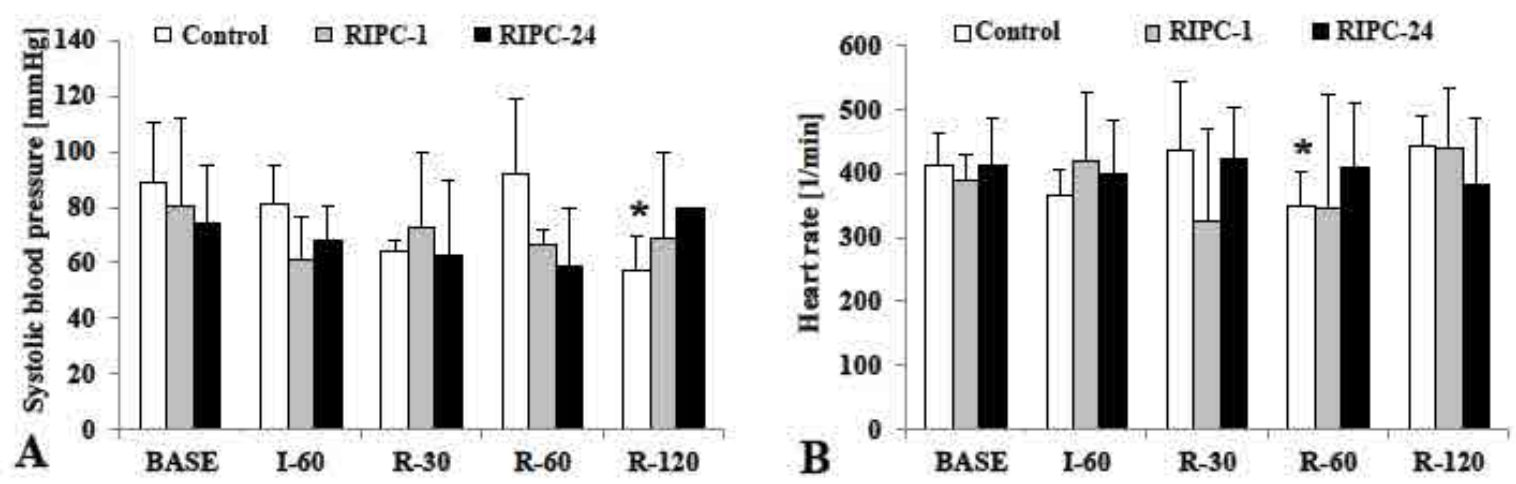

Figure 1 - Changes of systolic blood pressure $[\mathrm{mmHg}]$ and heart rate $[1 / \mathrm{min}]$ in the Control, the Remote ischemic preconditioning 1 hour (RIPC-1), and the Remote ischemic preconditioning 24 hour (RIPC-24) groups. Systolic blood pressure (A), Heart rate (B). Base = before the operation; $I-60=$ the end of the ischemia; $R 30=$ the 30th minute of the reperfusion; R-60 = the 60th minute of the reperfusion; $R-120=$ the 120 th minute of the reperfusion. Means \pm S.D.; ${ }^{*} p<0.05$ vs. Base.

\section{Respiratory rate}

There was no significant disparity in the changes of the respiratory rate, however a moderate elevation was observed in the Control and in the RIPC-24 during the reperfusion period of the process (Figure 2).

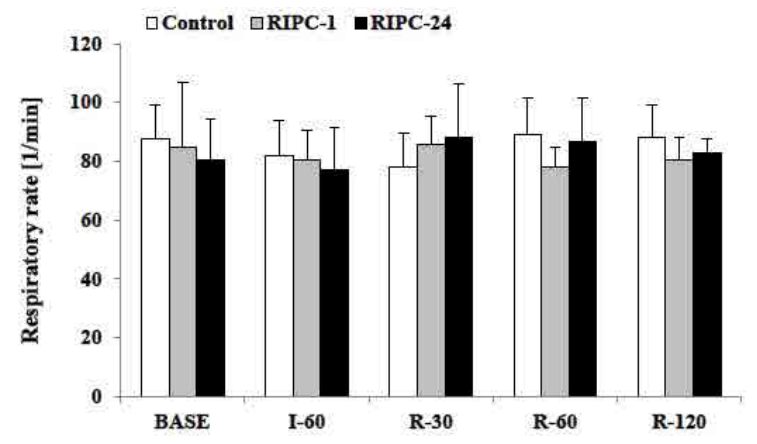

Figure 2 - Changes of respiratory rate $[1 / \mathrm{min}]$ in the Control, the Remote ischemic preconditioning 1 hour (RIPC-1), and the Remote ischemic preconditioning 24 hour (RIPC-24) groups. Base = before the operation; $I-60$ = the end of the ischemia; $\mathrm{R}-30=$ the 30th minute of the reperfusion; $\mathrm{R}-60=$ the 60th minute of the reperfusion; R-120 = the 120 th minute of the reperfusion. Means \pm S.D. 


\section{Rectal and liver surface temperature}

The measured rectal temperature values remained constant in all groups in the different time-points of the process (Figure 3).

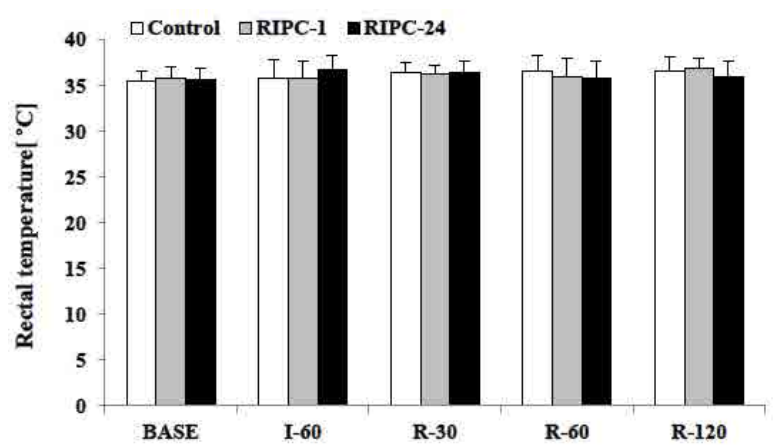

Figure 3 - Changes of rectal temperature $\left[\mathrm{C}^{\circ}\right]$ in the Control, the Remote ischemic preconditioning 1 hour (RIPC-1), and the Remote ischemic preconditioning 24 hour (RIPC-24) groups. Base = before the operation; $I-60$ = the end of the ischemia; $\mathrm{R} 30=$ the 30th minute of the reperfusion; R-60 = the 60th minute of the reperfusion; $\mathrm{R}-120=$ the 120 th minute of the reperfusion. Means \pm S.D.
Analysing the surface temperatures of the ischemic and non-ischemic part of the liver it can be established, that the temperature of the non-ischemic lobe elevated in the Control and in the RIPC-1 group at the end of the reperfusion (Control vs. Base $p=0.049$, RIPC- 1 vs. Base $p=0.012$ ). In the RIPC-24 group the values increased significantly at the $60^{\text {th }}$ minute of the reperfusion (RIPC-24 vs. Base $p=0.002$ ). Comparing the experimental groups the same range of alterations was found, significant intergroup difference was observed between the RIPC-24 and the Control at the $60^{\text {th }}$ minute of the reperfusion $(p=0.032 v s$. Control). The temperature data of the ischemic liver were similar to the non-ischemic liver and the same tendency of changes were observed, although the difference was significant in the Control group at the $30^{\text {th }}$ minute of the reperfusion $(p=0.015$ vs. Non-ischemic liver) (Figure 4).
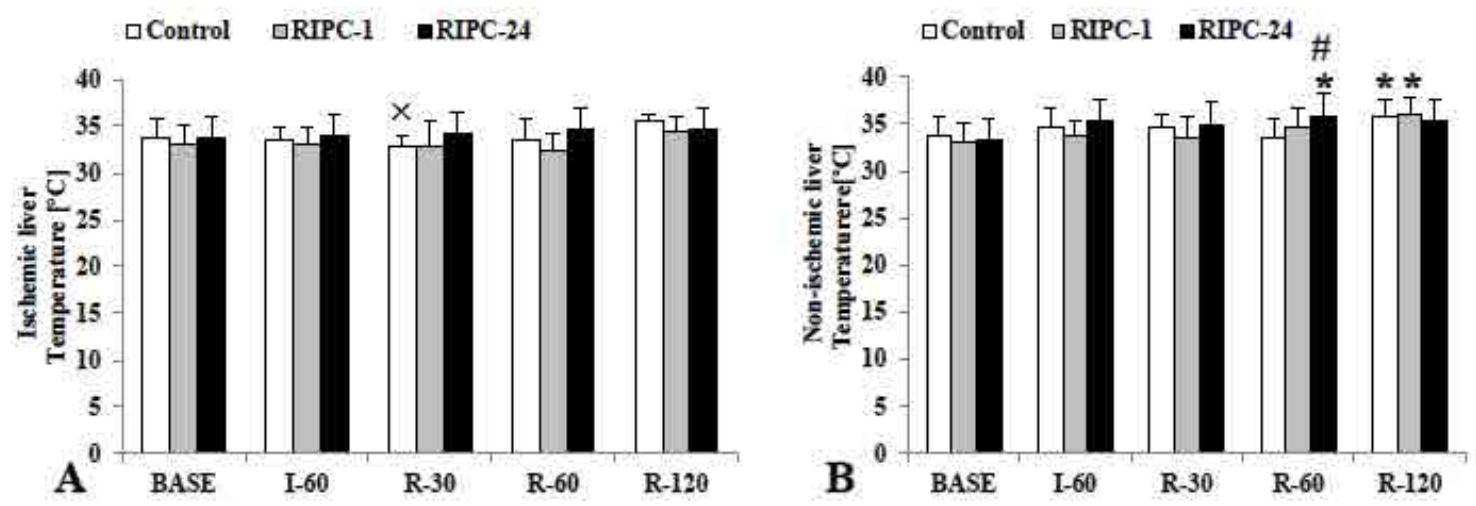

Figure 4 - Changes of liver surface temperature $\left[\mathrm{C}^{\circ}\right]$ in the Control, the Remote ischemic preconditioning 1 hour (RIPC-1), and the Remote ischemic preconditioning 24 hour (RIPC-24) groups. Non-ischemic liver (A), Ischemic liver (B). Base $=$ before the operation; $I-60=$ the end of the ischemia; $R 30=$ the 30th minute of the reperfusion; $\mathrm{R}-60=$ the 60 th minute of the reperfusion; $\mathrm{R}-120=$ the 120 th minute of the reperfusion. Means \pm S.D.; ${ }^{*} p<0.05$ vs. Base; $\# p<0.05$ vs. Control; $\times p<0.05$ vs. Non-ischemic liver.

\section{Microcirculatory measurements}

With regard to the non-ischemic lobe of the liver the BFU values of the Control group did not show remarkable changes in the observed follow-up period. In the RIPC-1 group the values elevated moderately by the end of the ischemic period, but during the reperfusion decreased again. In the RIPC-24 group the BFU parameters continuously decreased till the $30^{\text {th }}$ minute of the reperfusion, where the difference was significant compared to the base and the Control group ( $p=0.021$ vs. Base; $p=0.036$ vs. Control), then increased again. 
Examining the BFU values of the ischemic part of the liver an identical pattern of alterations was seen before the operation and at the end of the ischemia. At R-30 the BFU level of RIPC-24 group was significantly decreased $(p=0.021$ vs. Base; $p=0.036$ vs. Control). By the end of the examined reperfusion period the values were moderately elevated in all groups.

The BFU values of the non-ischemic liver versus the ischemic lobe were significantly lower in the Control group at R120 ( $p=0.035$ $v s$. ischemic liver) In the RIPC-1 group the BFU values of the ischemic lobe were lower at R-60 compared to the non-ischemic part of the liver
( $p=0.043$ vs. Non-ischemic liver).

The BFU parameters of the small intestine were increased at I-60 in the Control and in the RIPC-24 groups, then decreased during the reperfusion. At R-60 the difference was significant between the Control and RIPC1 groups ( $p=0.038 v$ s. Control).

With regard to the microcirculation of the right kidney consistently higher values were registered in the RIPC-1 group compared to the RIPC-24 group. In the RIPC-24 group the changes were significant versus Control at 160 and R60 ( $p=0.001$ and $p=0.010$ vs. Control) (Figure 5).
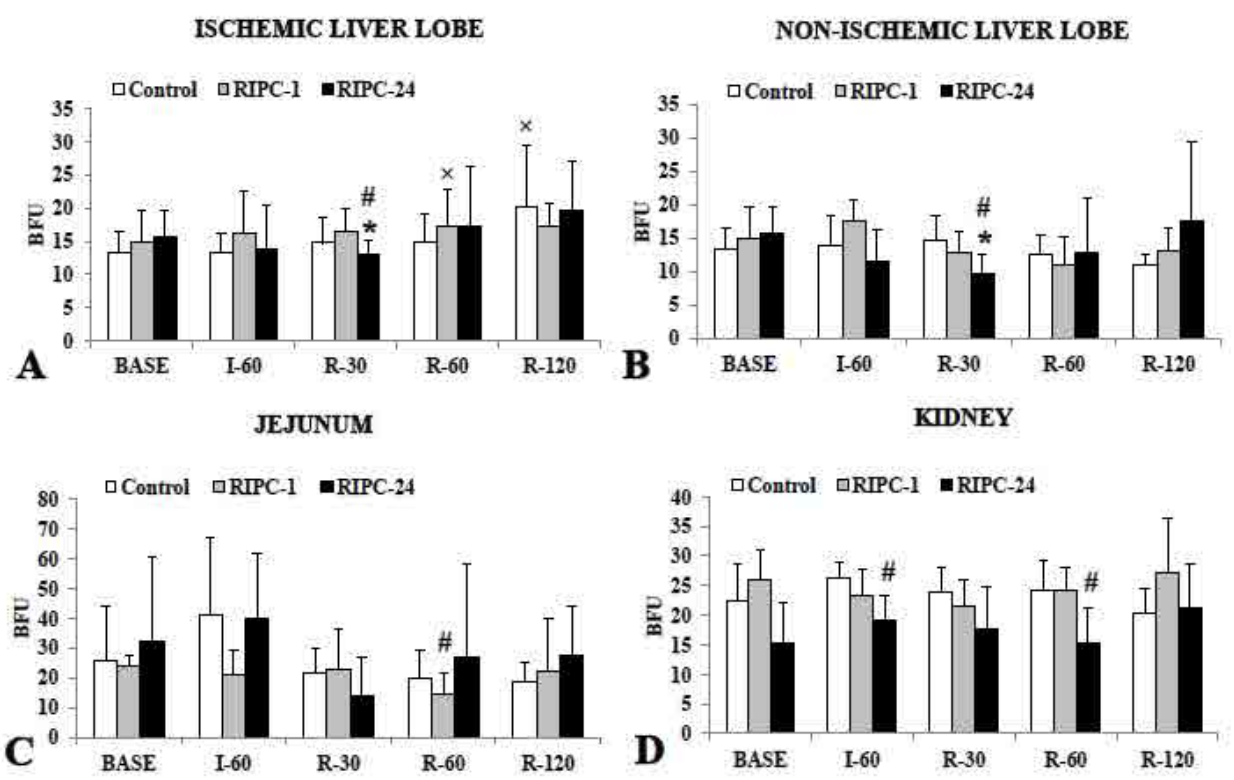

Figure 5 - Changes of the BFU values in the Control, the Remote ischemic preconditioning 1 hour (RIPC-1), and the Remote ischemic preconditioning 24 hour (RIPC-24) groups. Non-ischemic liver (A), Ischemic liver (B), Intestine (C), Kidney (D). Base = before the operation; I-60 = the end of the ischemia; R30 = the 30th minute of the reperfusion; R-60 = the 60th minute of the reperfusion; R-120 = the 120 th minute of the reperfusion. Means \pm S.D.; ${ }^{*}<<0.05$ vs. Base; $\# p<0.05$ vs. Control; $\times p<0.05$ vs. Non-ischemic liver.

\section{Histopathological examinations}

In our current study the following histological alterations were observed after ischemia-reperfusion injury (Figure 6): signs of sinusoidal congestion, pericentral desorganisation, necrosis in the pericentral region, hepatocyte dilatation, signs of inflammation in the central zone the cytoplasm is more eosinophil and homogenous. Quantifying the observed histological alterations of the ischemic lobe of the liver moderate sinusoidal congestion, necrosis and in $50 \%$ of the examined samples mild vacuolization could be detected in the sections of the Control group. Regarding the results of 
the RIPC-1 group it can be determined that the deterioration of the tissue damage was more serious compared to the Control group, as higher amount of hepatocyte necrosis close to the sinusoids, congestion and also inflammatory cells were described. Similar histopathological changes were found in the RIPC-24 group, but the vacuolization was more severe and even the total score was higher compared to the Control and the RIPC-1 group (Control: 4.125 \pm 1.96 ; RIPC-1: 5.83 \pm 1.6 ; RIPC24: 6.13 \pm 2.12 ).
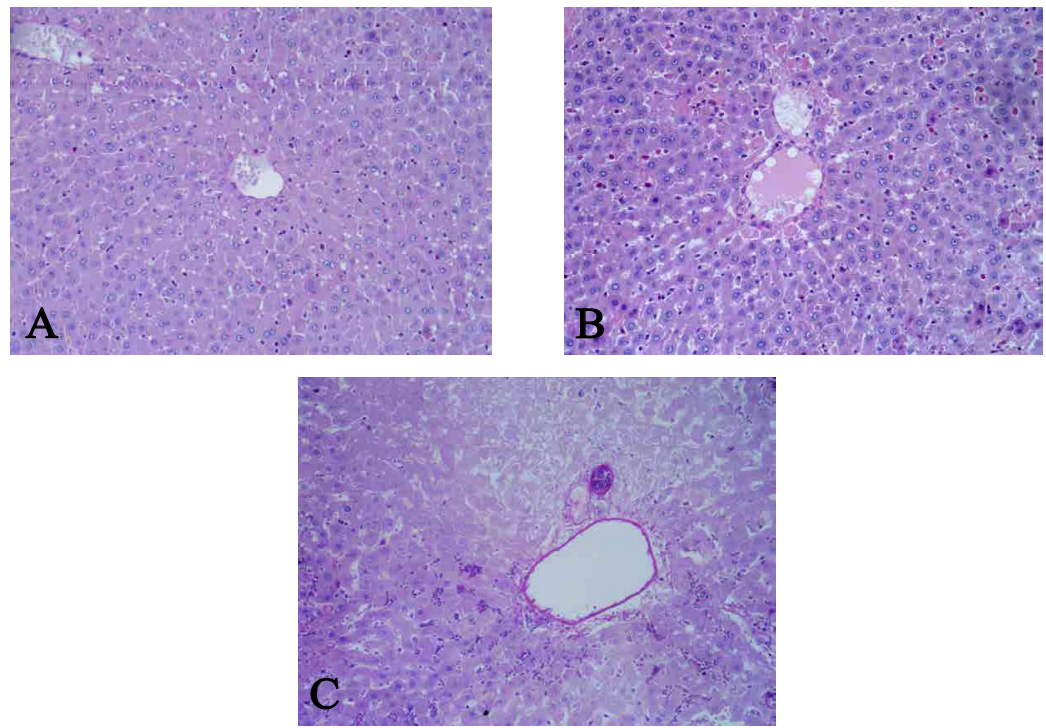

Figure 6 - Histology of the ischemic lobe of the liver (H \& E staining; original magnification: 20x). Samples from the Control (A), Remote ischemic preconditioning 1 hour before I/R (RIPC-1) (B), and Remote ischemic preconditioning 24 hour before I/R (RIPC-24) (C) groups.

\section{- Discussion}

The purpose of our present study was to compare the effect of early and late remote ischemic preconditioning protocols on hepatic ischemia-reperfusion injury by evaluating the hemodynamic, microcirculatory and histological changes.

Ischemia-reperfusion of the liver inevitably occurs during liver surgery by applying the Pringle manoeuvre, resulting in insufficiency of the liver remnant, associated with increased morbidity and mortality. Several surgical strategies have been developed and examined to reduce $I / R$ injury including remote ischemic preconditioning. Lai et al. ${ }^{24}$ proved the effectivity of RIPC in rat liver model in 2006. Numerous studies have been designed to reveal the exact mechanism, pathways and mediators of preconditioning, but a clear understanding is still lacking. There is also no consensus on the most effective timing of IPC protocols. Total duration, number of cycles, tissue volume, duration of the ischemic and reperfusion periods may all influence the outcome ${ }^{25}$.

The optimal interval between the preconditioning stimulus and the onset of sustained ischemia is also not known. In respect of coronary vessels it was concluded that if this interval exceeds 2 hours, the beneficial effects begin to wane. If extended to 24-72 hours, a 'second window of protection' emerges ${ }^{26}$. Another study found that in cardiac surgery the effect of RIPC was better if used $24 \mathrm{~h}$ before the ischemic insult ${ }^{27}$.

In our research RIPC was performed with the most commonly used protocol of 
3 cycles of 10-minute ischemia/10-minute reperfusion performed 1 or 24 hours before introducing partial hepatic ischemia ${ }^{28}$.

Concerning the hemodynamic parameters the alterations in the systolic blood pressure and the heart rate were steadier in RIPC groups compared to the control group. The heart rate was stable in RIPC-24 group, while it was rather fluctuating in RIPC- 1 and $C$ groups throughout the time course of observation. These findings partly differ from that of Tapuria et al. ${ }^{29}$. In their research a transient drop in $\mathrm{BP}$ with recovery to baseline by $R 30 / R 60$ in both I/R and RIPC groups was observed.

Neither the respiratory rate nor the rectal temperature showed significant changes during the observation period. The liver surface temperature values followed a similar pattern in all groups showing a moderate elevation that reached significance $v s$. base in RIPC-24 at R60 and in RIPC-1 and C groups by R120.

To monitor the microcirculation we used laser Doppler flowmetry. It is a widely used, non-invasive method, although having some drawbacks. The most important one is that several factors may influence the result including temperature, instability of the probe, organ movements, evaporisation of the tissue, local blood pressure and oxygenation ${ }^{30}$.

We found that by the end of the observed period the BFU values reached or exceeded the base values in both RIPC groups. The 1-hour preconditioning protocol could be more effective in preserving the microcirculation of the liver as in most of the examined time points higher BFU levels were registered in RIPC-1 than in RIPC-24 group. However, by the end of the $I / R$ period the highest BFU values were observed in the RIPC24 group.

Several studies demonstrated that RIPC protects the liver from IRI by modulation of the hepatic microcirculation. This protective effect is thought to be the result of increased RBC velocity, sinusoidal perfusion and decreased neutrophil adhesion and cell death, though the underlying mechanism is still not understood $^{28,31}$.

Tapuria et al..$^{29}$ found using intravital videoflourescence microscopy that RIPC had a positive effect on microcirculation as it resulted in an increased velocity of RBC flow, sinusoidal flow and sinusoidal perfusion compared to $\mathrm{I} / \mathrm{R}$ group at all time points. The significant increase in sinusoidal flow was due to increase in diameter as had been demonstrated by the author in earlier studies as well ${ }^{31,32}$.

It is known that micro-rheological parameters such as red blood cell deformability and aggregation play important role in influencing microcirculation ${ }^{22}$. In our former manuscript using the same model we reported the effects of RIPC on micro-rheological parameters. It was found that erythrocyte deformability was improved in the RIPC-1 group that supports the present findings.

Histologically endothelial cell disruption, cellular swelling, vacuolization, neutrophil infiltration and hepatocellular necrosis characterize the hepatic I/R injury. ${ }^{1}$

Freitas et al. ${ }^{33}$ examined various ischemia-reperfusion periods using Pringle manoeuvre (10-, 20- and 30-minute ischemia followed by 15-, 30-, 60- and 120-minute reperfusion) in rats and found that the longer the duration of ischemia and reperfusion, the greater are the morphologic changes'. Accordingly, 60-minute ischemia followed by 12-minute reperfusion employed in our study was expected to cause significant histological alterations.

Unexpectedly, these signs were more pronounced in both RIPC groups compared to the Control group. These results are against the findings of others, where the pathological changes in the RIPC groups were milder than those in the $\mathrm{I} / \mathrm{R}$ group ${ }^{29,34}$. The result of the Suzuki's score system were consistent to these observations. A possible reading of the controversial histological findings is the 
influence of acute phase reactions that is more pronounced in the RIPC-24 group due to the longer time period. The findings of Vega et al. ${ }^{35}$ provide further explanation. They have shown that xanthin-oxidase released from the ischemic hind limb during reperfusion is taken up by the liver where it mediates Kupffer-cell and polymorphonuclear leukocyte activation and thus contributes to the development of multiple organ failure.

A limitation of our study is that only healthy, young animals were exposed to ischemia-reperfusion. Based on the literature it is known that old age, steatotic or cirrhotic livers and certain co-morbidities are aggravating factors in ischemia-reperfusion injury ${ }^{9}$.

Limani et al. ${ }^{36}$ used a mice model for testing whether RIPC is an effective preventive strategy against I/R injury of an elderly liver. Their findings demonstrate that RIPC was superior to other preventing strategies, because the standard ischemic preconditioning method seemed to be ineffective in the case of old liver. Furthermore it was found that RIPC is better in promoting protective signalling, VEGF is the key mediator of protective RIPC effects in elderly liver and also reduces age-related deficiencies in hepatic sinusoids.

RIPC is an inexpensive, non-invasive, easily applicable method in different clinical situations. This method could be applied in patients undergoing liver surgery, therefore the examination of the effect of different RIPC protocols may serve further information in better understanding the pathophysiology of the process and finding the optimal way and the most effective method for clinical application to diminish the damage caused by I/R injury.

\section{Conclusions}

Regarding the hemodynamic and microcirculatory alterations, both early and late remote preconditioning protocols had beneficial effect. However, the histopathological findings were paradox. Based on our results it cannot be determined which RIPC protocol provides more protection against ischemiareperfusion injury in this rat model.

\section{References}

1. Kim YI. Ischemia-reperfusion injury of the human liver during hepatic resection. J Hepatobiliary Pancreat Surg. 2003;10:1959. PMID: 14605975.

2. van de Poll MCG, Derikx JP, Buurman WA, Peters WH, Roelofs HM, Wigmore SJ, Dejong $\mathrm{CH}$. Liver manipulation causes hepatocyte injury and precedes systemic inflammation in patients undergoing liver resection. World J Surg 2007;31:2033-8. PMID: 17668263.

3. Dello SAWG, Reisinger KW, van Dam RM, Bemelmans MHA, van Kuppevelt $\mathrm{TH}$, van den Broek MA, Olde Damink SW, Poeze M, Buurman WA, Dejong CHC. Total intermittent Pringle maneuver during liver resection can induce intestinal epithelial cell damage and endotoxemia. PLos One 2012;7(1):1-7. PMID: 22291982.

4. Miranda LEC, Viaro F, Caneviva R, Evora PRB. The experimental basis of hepatic ischemia-reperfusion injury: review. Acta Cir Bras. 2004;19:1-10. doi: 10.1590/S010286502004000100001.

5. Kupiec-Weglinski JW, Busuttil RW. Ischemia and reperfusion injury in liver transplantation. Transplant Proc. 2005;37(4):1653-6. PMID: 15919422.

6. Menger MD, Richter S, Yamauchi J, Vollmar B. Role of microcirculation in hepatic ischemia/ reperfusion injury. Hepatogastroenterology. 1999;46(Suppl 2):1452-7. PMID: 10431706.

7. Gurusamy KS, Gonzalez HD, Davidson BR. Current protective strategies in liver surgery. World J Gastroenterol. 2010;16(48):6098103. PMID: 21182224.

8. Jaeschke $H$, Woolbright BL. Current strategies to minimize hepatic ischemiareperfusion injury by targeting reactive oxygen species. Transplant Rev (Orlando, Fla) 2012;26(2):103-14. PMID: 22459037.

9. Bahde R, Spiegel H. Hepatic ischaemiareperfusion injury from bench to bedside. $\mathrm{Br} J$ Surg. 2010;97(10):1461-75. PMID: 20645395. 
10.Kim Yl, Fujita S, Hwang YJ, Nagase Y. One hundred hepatectomies without hospital death under intermittent hepatic inflow clamping for 30 minute duration. Hepatogastroenterology. 2014;61(131):5903. PMID: 26176041.

11.Heizmann O, Meimarakis $G$, Volk $A$, Matz D, Oertli D, Schauer RJ. Ischemic preconditioning induced hyperperfusion correlates with hepatoprotection after liver resection. World J Gastroenterol. 2010;16(15):1871-8. PMID: 20397265.

12.Murry $C E$, Jennings RB, Reimer $K A$. Preconditioning with ischemia: a delay of lethal cell injury in ischemic myocardium. Circulation. 1986;74:1124-36. PMID: 3769170.

13.Tapuria N, Kumar Y, Habib MM, Abu Amara M, Seifalian AM, Davidson BR. Remote ischemic preconditioning: a novel protective method from ischemia-reperfusion injury - a review. J Surg Res. 2008;150(2):304-30. PMID: 19040966.

14.Przyklenk K, Bauer B, Ovize M, Kloner RA, Whittaker P. Regional ischemic 'preconditioning' protects remote virgin myocardium from subsequent sustained coronary occlusion. Circulation. 1993;87:893-9. PMID: 7680290.

15.Kanoria S, Jalan R, Davies NA, Seifalian AM, Williams R, Davidson BR. Remote ischemic preconditioning of the hind limb reduces experimental liver warm ischaemia reperfusion injury. Br J Surg. 2006;93(6):7628. PMID: 16609953.

16.Kharbanda RK, Mortensen UM, White PA, Kristiansen SB, Schmidt MR, Hoschtitzky $J A$, Vogel $M$, Sorensen $K$, Redington AN, MacAllister R. Transient limb ischemia induces remote ischemic preconditioning in vivo. Circulation. 2002;106:2881-3. PMID: 12460865.

17.Waldow T, Alexiou K, Witt W, Albrecht $S$, Wagner $F$, Knaut $M$, Matschke $K$. Protection against acute porcine lung ischemia/reperfusion injury by systemic preconditioning via hind limb ischemia. Transpl Int. 2005;18:198-205. PMID: 15691273.

18.Jiménez-Castro $M B$, Elias-Miró $M$, CasillasRamirez A, Peralta C. Experimental models in liver surgery. In: Hesham Abdeldayem, Hepatic Surgery. 2013. p.121-66. doi:
$10.5772 / 51829$.

19.Szijarto A, Hahn O, Lotz G, Schaff Z, Madarász E, Kupcsulik PK. Effect of ischemic preconditioning on rat liver microcirculation monitored with laser Doppler flowmetry. J Surg Res. 2006;131(1):150-7. PMID: 16256139.

20. Kupcsulik P, Kokas P. Ischemic damage of the liver. Part II: In vivo investigation of the prevention of the ischemic lesion of the liver. Acta Hepatogastroenterol (Stuttg). 1979;26(4):284-9. PMID: 495025.

21.Leahy MJ, de Mul FF, Nilsson GE, Maniewski R. Principles and practice of the laserDoppler perfusion technique. Technol Health Care. 1999;7(2-3):143-62. PMID: 10463304.

22. Nemeth N, Szabo A. Microcirculation. In: Huifang C, Martins P (Eds.) Advances in experimental surgery. Hauppauge (NY): Nova Science Publishers; 2018. p.317-57.

23.Suzuki H, Robinson MK. Glutathione deficiency accentues hepatocellular fluid accumulation after ischemia-reperfusion. J Surg Res. 1994;57:632-9. PMID: 7967600.

24.Lai IR, Chang KJ, Chen CF, Tsai HW. Transient limb ischemia induces remote preconditioning in liver among rats: the protective role of heme oxygenase-1. Transplantation. 2006;81:1311-7. PMID: 16699460.

25.Galagudza MM, Sonin DL, Vlasov TD, Kurapeev DI, Shlyakhto EV. Remote vs. local ischaemic preconditioning in the rat heart: infarct limitation, suppression of ischaemic arrhythmia and the role of reactive oxygen species. Int J Exp Pathol. 2016;97(1):66-74. PMID: 26990944.

26. Przyklenk K. Reduction of myocardial infarct size with ischemic 'conditioning': Physiologic and technical considerations. Anesth Analg. 2013;117:891-901. PMID: 23960036.

27.Cheung MM, Kharbanda RK, Konstantinov IE, Shimizu M, Frndova H, Li J, Holtby HM, Cox PN, Smallhorn JF, Van Arsdell GS, Redington AN. Randomized controlled trial of the effects of remote ischemic preconditioning on children undergoing cardiac surgery: first clinical application in humans. J Am Coll Cardiol. 2006;47(11):2277-82. PMID: 16750696.

28. Kanoria S, Robertson FP, Mehta NN, Fusai G, Sharma D, Davidson BR. Effect of remote 
ischaemic preconditioning on liver injury in patients undergoing major hepatectomy for colorectal liver metastasis: a pilot randomised controlled feasibility trial. World J Surg. 2017;41(5):1322-30. PMID: 27933431.

29.Tapuria N, Junnarkar SP, Abu-Amara M, Fuller B, Seifalian AM, Davidson BR. Haemoxygenase modulates cytokine induced neutrophil chemoattractant in hepatic ischemia reperfusion injury. World J Gastroenterol. 2016;22(33):7518-35. PMID: 27672274.

30.Obeid AN, Barnett NJ, Dougherty G, Ward G. A critical review of laser Doppler flowmetry. J Med Eng Technol. 1990;14(5):178-81. PMID: 2231661.

31.Tapuria N, Junnarkar SP, Dutt N, Abu-Amara $M$, Fuller B, Seifalian AM, Davidson BR. Effect of remote ischemic preconditioning on hepatic microcirculation and function in a rat model of hepatic ischemia reperfusion injury. HPB. 2009;11(2):108-17. PMID: 19590633.

32.Tapuria N, Junnarkar S, Abu-Amara M, Fuller $B$, Seifalian AM, Davidson BR. Modulation of microcirculatory changes in the late phase of hepatic ischaemia-reperfusion injury by remote ischaemic preconditioning. HPB (Oxford). 2012;14:87-97. PMID: 22221569.

33.Freitas SH, Doria RGS, Bueno RS, Rocha WB, Filho JRE, Vidane AS, Ambrósio CE. Evaluation of potential changes in liver and lung tissue of rats in an ischemia-reperfusion injury model (modified Pringle maneuver). PLoS One. 2017 Jun 12;12(6):e0178665. PMID: 28604841.

34.Duan YF, An Y, Zhu F, Jiang Y. Remote ischemic preconditioning protects liver ischemiareperfusion injury by regulating eNOS-NO pathway and liver microRNA expressions in fatty liver rats. Hepatobiliary Pancreat Dis Int. 2017;16(4):387-94. PMID: 28823369.

35.Vega VL, Mardones L, Maldonado M, Nicovani S, Manríquez V, Roa J, Ward PH. Xanthine oxidase released from reperfused hind limbs mediate Kupffer cell activation, neutrophil sequestration, and hepatic oxidative stress in rats subjected to tourniquet shock. Shock. 2000;14(5):56571. PMID: 11092691.

36.Limani $P$, Linecker $M$, Oberkofler CE, Barmettler G, Kaech A, Graf R, Humar B, ClavienPA. Remoteischemicpreconditioning: A novel strategy in rescuing older livers from ischemia-reperfusion injury in a rodent model. Ann Surg. 2016;264(5):797-803. PMID: 27584570.

\section{- Acknowledgement}

The technical and laboratory staff, Department of Operative Techniques and Surgical Research, Faculty of Medicine, University of Debrecen.

\section{Correspondence:}

Norbert Nemeth

Department of Operative Techniques and Surgical Research

Institute of Surgery, Faculty of Medicine, University of Debrecen

H-4032 Debrecen, Nagyerdei krt. 98 Hungary

Phone/Fax: +36-52-416-915

nemeth@med.unideb.hu

Received: Mar 12, 2018

Review: May 10, 2018

Accepted: June 09, 2018
Conflict of interest: none

Financial source: Bridging Fund, Faculty of Medicine, University of Debrecen
${ }^{1}$ Research performed at Department of Operative Techniques and Surgical Research, Institute of Surgery, Faculty of Medicine, University of Debrecen, Hungary. 